\title{
Filtering of false positive microRNA candidates by a clustering-based approach Wing-Sze Leung ${ }^{1}$, Marie CM Lin ${ }^{2}$, David W Cheung ${ }^{1}$ and SM Yiu*1
}

\author{
Address: ${ }^{1}$ Department of Computer Science, The University of Hong Kong, Pokfulam Road, Hong Kong and ${ }^{2}$ Department of Chemistry, Open \\ Laboratory of Chemical Biology, The University of Hong Kong, Pokfulam Road, Hong Kong \\ Email: Wing-Sze Leung - h0328078@hkusua.hku.hk; Marie CM Lin - mcllin@hkusua.hku.hk; David W Cheung - dcheung@cs.hku.hk; \\ SM Yiu* - smyiu@cs.hku.hk \\ * Corresponding author
}

from Asia Pacific Bioinformatics Network (APBioNet) Seventh International Conference on Bioinformatics (InCoB2008)

Taipei, Taiwan. 20-23 October 2008

Published: 12 December 2008

BMC Bioinformatics 2008, 9(SuppI I2):S3 doi:I0.II86/I47I-2I05-9-SI2-S3

This article is available from: http://www.biomedcentral.com/I47I-2I05/9/SI2/S3

(C) 2008 Leung et al; licensee BioMed Central Ltd.

This is an open access article distributed under the terms of the Creative Commons Attribution License (http://creativecommons.org/licenses/by/2.0), which permits unrestricted use, distribution, and reproduction in any medium, provided the original work is properly cited.

\begin{abstract}
Background: MicroRNAs are small non-coding RNA gene products that play diversified roles from species to species. The explosive growth of microRNA researches in recent years proves the importance of microRNAs in the biological system and it is believed that microRNAs have valuable therapeutic potentials in human diseases. Continual efforts are therefore required to locate and verify the unknown microRNAs in various genomes. As many miRNAs are found to be arranged in clusters, meaning that they are in close proximity with their neighboring miRNAs, we are interested in utilizing the concept of microRNA clustering and applying it in microRNA computational prediction.

Results: We first validate the microRNA clustering phenomenon in the human, mouse and rat genomes. There are $45.45 \%, 51.86 \%$ and $48.67 \%$ of the total miRNAs that are clustered in the three genomes, respectively. We then conduct sequence and secondary structure similarity analyses among clustered miRNAs, non-clustered miRNAs, neighboring sequences of clustered miRNAs and random sequences, and find that clustered miRNAs are structurally more similar to one another, and the RNAdistance score can be used to assess the structural similarity between two sequences. We therefore design a clustering-based approach which utilizes this observation to filter false positives from a list of candidates generated by a selected microRNA prediction program, and successfully raise the positive predictive value by a considerable amount ranging from $15.23 \%$ to $23.19 \%$ in the human, mouse and rat genomes, while keeping a reasonably high sensitivity.

Conclusion: Our clustering-based approach is able to increase the effectiveness of currently available microRNA prediction program by raising the positive predictive value while maintaining a high sensitivity, and hence can serve as a filtering step. We believe that it is worthwhile to carry out further experiments and tests with our approach using data from other genomes and other prediction software tools. Better results may be achieved with fine-tuning of parameters.
\end{abstract}




\section{Background}

MicroRNAs (miRNAs) are small non-coding RNA gene products of 19-25 nucleotides (nt) long, which function to repress the translation or mediate the degradation of their target mRNAs. A 22 nt mature miRNA is derived from a precursor transcript of 60-80 nt in length, which is named as pre-miRNA. Pre-miRNAs can potentially fold into a hairpin structure without large internal loops or bulges.

MiRNAs were found to play diversified roles from species to species $[1,2]$. In recent years, researches on the roles of miRNAs in cancers have been increasing tremendously, and miRNAs are suggested to have important therapeutic potential in human diseases. To date, there are 678, 472 and 287 miRNA entries for the human, mouse and rat genomes deposited in miRBase $[3,4]$, the home of miRNA data on the web, in Release 11.0, respectively. Yet some studies suggested that the total number of miRNAs existing in a vertebrate genome can reach at least $800[5,6]$, therefore continual efforts should be made on locating and verifying the unknown miRNAs. A number of computational prediction methods and software tools have been developed over the years for this purpose [7], however the datasets adopted by the various prediction tools are different and older methods are usually outweighed by the newly developed ones in terms of specificity and sensitivity.
In this paper, we first describe and validate the clustering phenomenon of miRNAs in the human, mouse and rat genomes by computational means. We then develop a clustering-based approach to a selected software tool, ProMirII-g [8,9], which was launched in 2006, aiming to filter their false positive miRNA predictions.

\section{Results and discussion MiRNA clustering}

Many miRNAs are found to be arranged in clusters [10], meaning that they are in close proximity with their neighboring miRNAs. MiRNAs located in the same cluster are usually co-regulated and co-expressed $[11,12]$, and recent studies suggest that miRNA clusters play important biological roles in specific tissues or genomes. Examples include cell proliferation in human lung cancer tissues [13], latent and lytic replication of Kaposi's sarcoma-associated herpesvirus [14], testis development and spermatogenesis in primates [15].

In view of this, we believe that miRNA clustering can be used to assist the prediction of novel miRNAs, and here we analyze how this idea can be applied computationally.

\section{Analysis of miRNA clustering in the human, mouse and rat genomes}

The definition of a miRNA cluster varies among researchers. Altuvia and colleagues defines a cluster in which there

Table I: Effects on the distance of chromosomal separation of clustered miRNAs on the number of clustered miRNAs found in the human, mouse and rat genomes.

\begin{tabular}{|c|c|c|c|c|c|c|c|}
\hline \multirow[t]{2}{*}{ Genome } & \multirow[t]{2}{*}{ Effect } & \multicolumn{6}{|c|}{ Chromosomal distance at which two clustered miRNAs are separated } \\
\hline & & $1500 \mathrm{nt}$ & $3000 \mathrm{nt}$ & $6000 \mathrm{nt}$ & $10000 \mathrm{nt}$ & $25000 \mathrm{nt}$ & $50000 \mathrm{nt}$ \\
\hline \multirow[t]{3}{*}{ Human } & \# of clustered miRNAs & 196 & 217 & 240 & 241 & 242 & 261 \\
\hline & \# of clusters defined & 71 & 68 & 60 & 60 & 60 & 65 \\
\hline & Average cluster size & 2.76 & 3.19 & 4 & 4.02 & 4.03 & 4.02 \\
\hline \multirow[t]{3}{*}{ Mouse } & \# of clustered miRNAs & 204 & 215 & 237 & 243 & 253 & 260 \\
\hline & \# of clusters defined & 70 & 53 & 55 & 57 & 58 & 61 \\
\hline & Average cluster size & 2.91 & 4.06 & 4.31 & 4.26 & 4.36 & 4.26 \\
\hline \multirow[t]{3}{*}{ Rat } & \# of clustered miRNAs & 119 & 129 & 145 & 154 & 154 & 160 \\
\hline & \# of clusters defined & 46 & 45 & 48 & 49 & 47 & 48 \\
\hline & Average cluster size & 2.59 & 2.87 & 3.04 & 3.14 & 3.28 & 3.14 \\
\hline
\end{tabular}

"Average cluster size" is equivalently to the average number of miRNAs found in a single cluster. It can be seen that there is an abrupt increase in the number of clustered miRNAs from the case of $3000 \mathrm{nt}$ to the case of $6000 \mathrm{nt}$. There are little effects on the number of clustered miRNAs and the number of clusters defined when the separation is more than $10000 \mathrm{nt}$. To conclude, among the six distances that we have tested, $6000 \mathrm{nt}$ is an optimal chromosomal distance bound within which two clustered miRNAs are separated. 


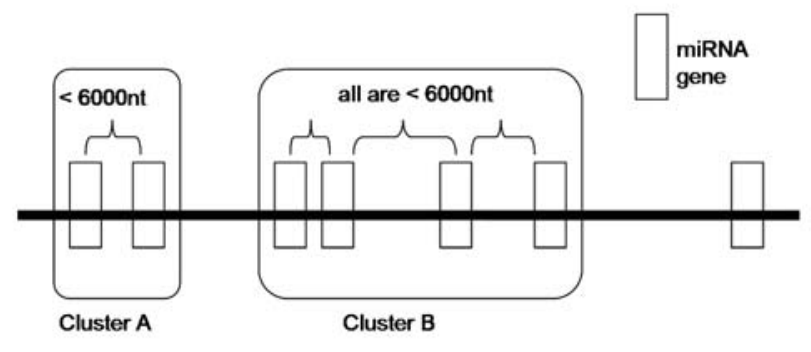

Figure I

Our definition of a cluster. MiRNAs which are separated by a distance of less than $6000 \mathrm{nt}$ are grouped as one cluster.

are two or more miRNA genes with pairwise chromosomal distances of at most $3000 \mathrm{nt}$ [10]. Weber [16] suggested the following criteria of a cluster: same orientation and not separated by a transcription unit or a miRNA in the opposite orientation. A microarray study reveals that an abrupt transition in the correlation between pairs of expressed miRNAs occurs at a distance of $50 \mathrm{~kb}$, implying that miRNAs separated by less than $50 \mathrm{~kb}$ typically derive from a common transcript [17]. In many other studies, the term 'cluster' is used without a proper and clear definition [18-20], and is also used to describe the phylogenetic relationships of miRNAs $[11,21]$. To assess the clustering property of miRNAs in the human, mouse and rat genomes, we want to have our own definition of a miRNA cluster. We define that two miRNAs belong to the same cluster if (1) they are located on the same strand of the same chromosome, i.e. same orientation; and (2) they are separated by a chromosomal distance of not more than $6000 \mathrm{nt}$. This distance of $6000 \mathrm{nt}$ is not arbitrary. We first choose six different distances, which are $1500 \mathrm{nt}$, $3000 \mathrm{nt}, 6000 \mathrm{nt}, 10000 \mathrm{nt}, 25000 \mathrm{nt}$ and $50000 \mathrm{nt}$, and then we test the effect of the distances on the number of clusters and the number of clustered miRNAs formed. As shown in Table 1, there is an abrupt increase in the number of clustered miRNAs from the case of $3000 \mathrm{nt}$ to the case of $6000 \mathrm{nt}$. There are little effects on the number of clustered miRNAs and the number of clusters defined when the separation is more than $10000 \mathrm{nt}$. To conclude, among the six distances that we have tested, $6000 \mathrm{nt}$ is an optimal chromosomal distance bound within which two clustered miRNAs are separated. Figure 1 illustrates our definition of a miRNA cluster and Table 2 summarizes our results of the miRNA clustering analyses. The human and mouse datasets used in this paper were downloaded from Release 10.0 of miRBase and the rat datasets were from Release 10.1 . There are $45.45 \%, 51.86 \%$ and $48.67 \%$ of the total miRNAs that are clustered in the human, mouse and rat genomes, respectively.
Table 2: The number of clustered miRNAs and isolated miRNAs found in the human, mouse and rat genomes using our definition of miRNA cluster.

\begin{tabular}{lllll}
\hline & & Human & Mouse & Rat \\
\hline Version of miRBase & & 10.0 & 10.0 & 10.1 \\
\hline Total \# of clusters & & 60 & 55 & 48 \\
\hline Size of clusters & Min & 2 & 2 & 2 \\
\hline & Mean & 4 & 4.31 & 3.04 \\
\hline Max & 43 & 52 & 17 \\
\hline Clustered miRNAs & $\#$ & 240 & 237 & 146 \\
\hline Total \# of miRNAs & & 288 & 220 & 154 \\
\hline
\end{tabular}

"Version of miRBase" denotes the update version of miRBase where the datasets are downloaded. "Size of clusters" is equivalently to the number of miRNAs found in a single cluster. There are 60,55 and 47 clusters identified in the three genomes respectively, which are equivalent to $45.45 \%, 51.86 \%$ and $48.67 \%$ of the total human, mouse and rat miRNAs.

\section{Similarity analyses among clustered miRNAs, non- clustered miRNAs, neighboring sequences of clustered miRNAs and random sequences}

As there are nearly half of the total miRNAs organized in clusters, we are interested in testing whether there are any relationships or similarities among them. We assess the sequence and secondary structure similarities among miRNAs in the same cluster by aligning the precursors of each clustered miRNA with the sequences from the following four categories in a pairwise manner:

(i) its fellow miRNAs found in the same cluster;

(ii) miRNAs located outside its cluster;

(iii) random sequences extracted from the genome; and

(iv) neighboring sequences extracted from its flanking $3000 \mathrm{nt}$ regions.

The software T-COFFEE [22] (Version 5.05) is used for pairwise sequence alignment. The program RNAdistance of the Vienna RNA package [23] (Version 1.7.1) is used to compute the distance between two miRNA secondary structures, which are determined by RNAfold. Figure 2 


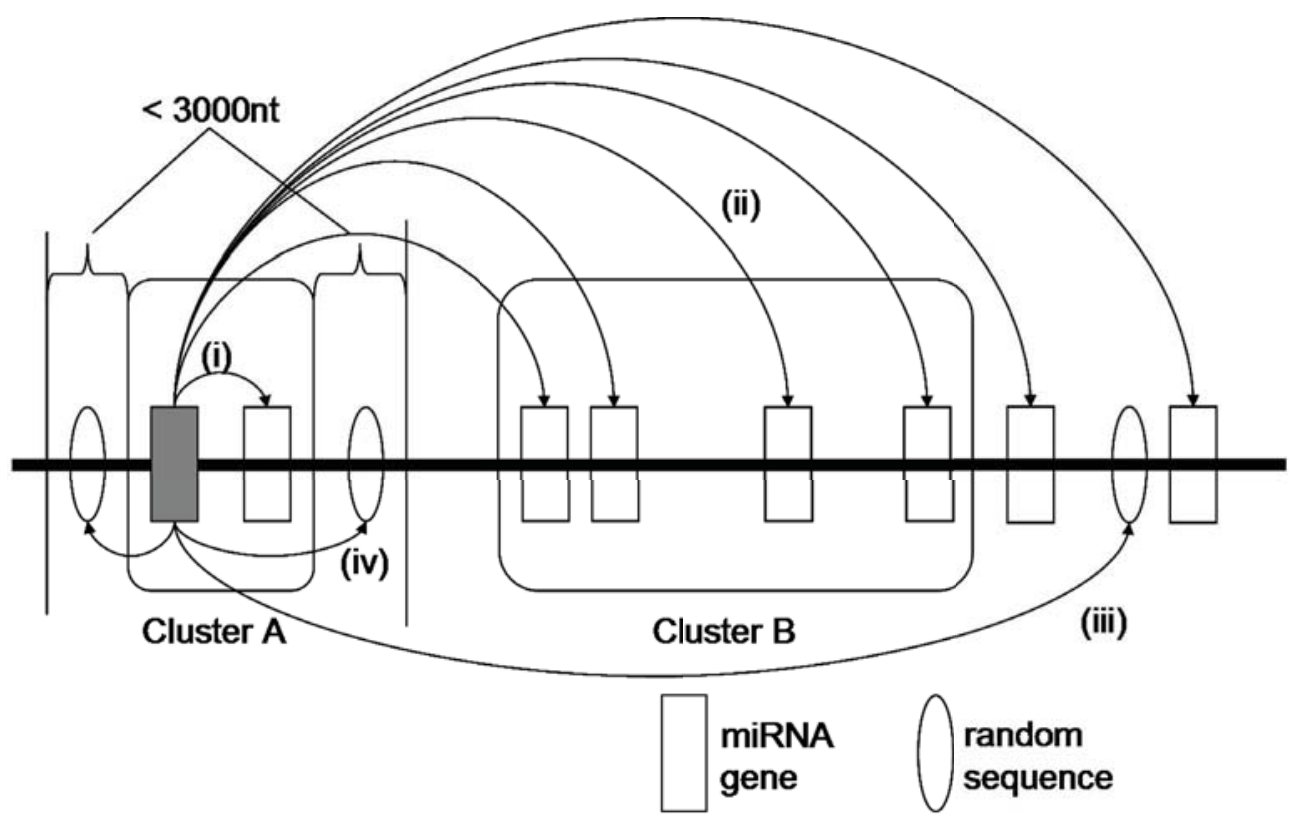

Figure 2

Similarity analyses of a clustered miRNA with four groups of sequences. A clustered miRNA is aligned with sequences from four categories: (i) miRNA(s) in the same cluster; (ii) miRNAs outside its cluster; (iii) random sequences extracted from the genome; and (iv) random sequences extracted from its flanking $3000 \mathrm{nt}$ region.

shows how a clustered miRNA is aligned to sequences of the four categories.

As shown in Table 3, there is no statistically significant difference among the sequence alignment scores of the four categories (t-test, p-value < 0.05), suggesting that sequence similarity is unlikely to be useful for identifying clustered miRNAs. Interestingly, the distance between the secondary structures of miRNAs located in the same cluster is found to be much smaller than the distance obtained by comparing the structures of clustered miRNAs with the sequences from the other three categories ( $\mathrm{t}$-test, $\mathrm{p}$-value < 0.0001 ). In other words, clustered miRNAs are structurally more similar to one another, and the RNAdistance score can be used to assess the structural similarity between two sequences. Based on this observation, we propose a clustering-based approach to improve the effectiveness of computational prediction of miRNAs.

\section{Performance analyses of ProMirll-g and miR-abela}

We select two software tools to test our proposed clustering-based approach, namely ProMirII-g [8,9] and miRabela [24]. In terms of positive predictive value (PPV) and sensitivity (SE), we first analyze the performances of these two prediction tools, and the results serve as a benchmark for comparison with our approach. Both ProMirII-g and miR-abela allow users to set a prediction threshold. Using a relaxed threshold, more true positives (TPs) and predic- tions will be obtained, yet at the same time a large number of false positives (FPs) will be included. In other words, a high SE and a low PPV are expected. Our approach aims at increasing the PPV by filtering as many as FPs as possible with the application of miRNA clustering.

Table 4 illustrates the results of the performance analyses. ProMirII-g works better than miR-abela on all the three genomes under our investigation, giving a SE ranging from $81.22 \%$ to $89.58 \%$. miR-abela, on the contrary, does not show satisfactory performance on the prediction of human, mouse and rat miRNAs, with only around $60 \%$ of SE achieved. Both of them produce a large number of false FPs along with the TPs, and therefore low PPVs are resulted with a range from $13.31 \%$ to $31.16 \%$. Clearly the prediction programs will be more useful and reliable if their FP rates can be reduced.

\section{Application of miRNA clustering: a clustering-based approach}

Recalling that clustered miRNAs are more structurally similar to one another as determined by the RNAdistance scores, we therefore design a clustering-based approach which utilizes this observation to filter false positives. The detailed steps of our approach are described in the section of Methods under the sub-title of "Our clustering-based approach" and Figure 3 provides the overview of the approach. 
Table 3: Results of the similarity analyses between clustered miRNAs and other sequences.

\begin{tabular}{|c|c|c|c|c|c|c|c|c|c|}
\hline \multirow{2}{*}{$\begin{array}{c}\text { Genome } \\
\text { Human }\end{array}$} & \multirow[b]{2}{*}{ Category } & \multicolumn{4}{|c|}{ T-COFFEE sequence alignment score } & \multicolumn{4}{|c|}{ RNAdistance structure alignment score } \\
\hline & & Maximum & Average & Minimum & Std Dev & Maximum & Average & Minimum & Std Dev \\
\hline & (i) & 79 & 45.20 & 17 & 15.70 & 71 & 28.86 & 0 & 9.73 \\
\hline & (ii) & 73 & 46.30 & 15 & 12.96 & 94 & 37.21 & 15 & 11.29 \\
\hline & (iii) & 74 & 45.47 & 17 & 14.13 & 203 & 140.89 & 104 & 18.78 \\
\hline & (iv) & 77 & 44.81 & 15 & 12.60 & 134 & 75.55 & 28 & 22.19 \\
\hline \multirow[t]{4}{*}{ Mouse } & (i) & 79 & 43.63 & 0 & 14.40 & 72 & 30.41 & 0 & 9.33 \\
\hline & (ii) & 78 & 45.28 & 16 & 13.03 & 69 & 35.19 & 10 & 9.67 \\
\hline & (iii) & 75 & 43.72 & 16 & 14.40 & 239 & 139.93 & 103 & $|7.3|$ \\
\hline & (iv) & 72 & 45.37 & 15 & 13.33 & 128 & 69.55 & 26 & 19.31 \\
\hline \multirow[t]{4}{*}{ Rat } & (i) & 75 & 44.28 & 17 & 12.83 & 113 & 31.41 & 11 & 12.60 \\
\hline & (ii) & 72 & 45.18 & 16 & 13.12 & 116 & 35.68 & 13 & 11.73 \\
\hline & (iii) & 69 & 45.03 & 16 & 12.48 & 198 & 143.12 & 110 & 18.43 \\
\hline & (iv) & 73 & 47.90 & 15 & 13.26 & 114 & 74.69 & 32 & 18.74 \\
\hline
\end{tabular}

Sequence and secondary structural alignments are performed for each clustered miRNA with sequences from the following categories: (i) clustered miRNAs, (ii) non-clustered miRNAs, (iii) random and (iv) neighboring sequences. A higher score implies a greater distance and hence a higher degree of dissimilarity. Std Dev, standard deviation.

From Table 5, it is clear that our approach is able to increase the PPV to a large extent, from $17.37 \%$ to $21.25 \%$. At the same time, it retains most TPs with just a slight drop of less than $10 \%$ in SE when it is applied to the human and the mouse genome. The approach appears to sacrifice the SE for the increase in PPV when it is tested on the rat genome. Since our filtering approach is based on miRNA clustering, it works best when the cluster has more than one TP. Table 6 shows a more suitable comparison when the clusters with less than two TPs are excluded from our test. The increase in PPV outweighs the drop in $\mathrm{SE}$ in all the three cases, suggesting that our approach is effective in filtering FPs without losing too many TPs.

\section{Conclusion}

In this paper, we first validate the phenomenon of miRNA clustering in the human, mouse and rat genomes and confirm that there are more than $45 \%$ of the miRNAs in these genomes which can form clusters. We demonstrate that the secondary structure of a clustered pre-miRNA is more similar to its neighbouring pre-miRNAs located in the same cluster, when compared to the sequences outside clusters. Using this property, we design a clustering-based approach to filter the FPs resulting from a miRNA prediction software named ProMirII-g and successfully raise the PPV by a considerable amount ranging from $15.23 \%$ to $23.19 \%$ in the human, mouse and rat genomes. At the same time, the approach is able to retain a reasonably high SE. In view of this, we conclude that our approach is shown to be effective in raising the PPV of a software tool, particularly in the human genomes. We believe that it is worthwhile to carry out further experiments and tests with our approach using data from other genomes and other prediction software tools. Better results may be achieved with fine-tuning of parameters.

\section{Methods}

Performance analyses of ProMirll-g and miR-abela

The following steps are applied to data from the genomes of human, mouse and rat respectively.

Step 1: A group of 10000 nt-long sequences are extracted from the genome as the input sequences to the prediction software. Each of the long sequences consists of the clustered miRNAs identified in the genomes as mentioned in the section of "Analysis of miRNA clustering in the 
Table 4: Results of the performance analyses of ProMirll-g and miR-abela using human, mouse and rat genome data.

\begin{tabular}{|c|c|c|c|c|c|}
\hline Software & Statistics & Human & Mouse (AII) & Mouse (Distinct) & Rat \\
\hline \multirow[t]{6}{*}{ ProMirll-g } & \# of predictions & 690 & 656 & 640 & 615 \\
\hline & \# of TPs & 215 & 199 & 183 & 127 \\
\hline & \# of FPs & 475 & 457 & 457 & 485 \\
\hline & \# of real miRNAs missed & 25 & 46 & 29 & 19 \\
\hline & SE & $89.58 \%$ & $81.22 \%$ & $85.92 \%$ & $86.99 \%$ \\
\hline & PPV & $31.16 \%$ & $30.34 \%$ & $28.59 \%$ & $20.65 \%$ \\
\hline \multirow[t]{6}{*}{ miR-abela } & \# of predictions & 1036 & 915 & 901 & 646 \\
\hline & \# of TPs & 149 & 140 & 126 & 86 \\
\hline & \# of FPs & 887 & 775 & 775 & 560 \\
\hline & \# of real miRNAs missed & 91 & 105 & 86 & 60 \\
\hline & SE & $62.08 \%$ & $57.14 \%$ & $59.43 \%$ & $58.90 \%$ \\
\hline & PPV & $14.38 \%$ & $15.30 \%$ & $13.98 \%$ & $13.31 \%$ \\
\hline
\end{tabular}

It should be noted that in the mouse genome, 25 of the pre-miRNAs are duplicated. In other words, only 212 mouse pre-miRNAs are distinct in the genome. To avoid overestimation of the performance of the software, we identify the duplicated ones and conduct two measurements. \#, number; FP, false positives; TP, true positives; SE, sensitivity; PPV, positive predictive value.

human, mouse and rat genomes". Large clusters which span over $10000 \mathrm{nt}$ are split into smaller clusters.

Step 2: Each sequence is inputted to ProMirII-g and miRabela. For ProMirII-g, 0.001 is selected as the prediction threshold (ProMiR value). For miR-abela, the prediction threshold is set at -10 . Other parameters are set by default. A list of outputs, representing the potential miRNA candidates, is generated.

Step 3: The outputs are checked against the clustered miRNAs found in the genomes. The output candidates which match the clustered miRNAs are the true positives, and the rest of the predicted candidates are the false positives.

With the total number of predictions, the total number of clustered miRNAs in the genome, the number of TPs and the number of FPs, we evaluate the performance of the two miRNA prediction tools in terms of the SE and PPV. The formulas for calculating SE and PPV are as follows:

$\mathrm{SE}=\mathrm{TP} /$ total number of clustered miRNAs in the genome $\mathrm{PPV}=\mathrm{TP} /$ total number of predictions, i.e. $\mathrm{TP} /(\mathrm{TP}+\mathrm{FP})$

Table 4 summarizes the results.

\section{Our clustering-based approach}

Stage I

A miRNA prediction program with prediction threshold set at a relaxed value is run with the same set of input sequences as described in the performance analyses of the prediction software. A list of candidates is produced, which are potential clustered miRNAs. Since the performance of miR-abela is not satisfactory, only ProMirII-g is used to test the effectiveness of our approach. 0.001 is chosen as the predictive value as it is the most relaxed threshold.

\section{Stage 2}

Pairwise structural alignment between each pair of candidates is conducted using RNAdistance. RNAdistance reads RNA secondary structures and calculates one or more measures for their dissimilarity, based on tree or string editing (alignment). Briefly, it first translates the RNA secondary structures, which is inputted by the user using the bracket format or coarse grained representations, into tree structures. The standard morphologic features like bulge, internal, multi-branch and hairpin loops are captured in the tree structures. It then aligns the trees using a multiple alignment program [25]. Since a higher RNAdistance score implies that the pair of candidate sequences have relatively different structures and vice versa, if a candidate has 


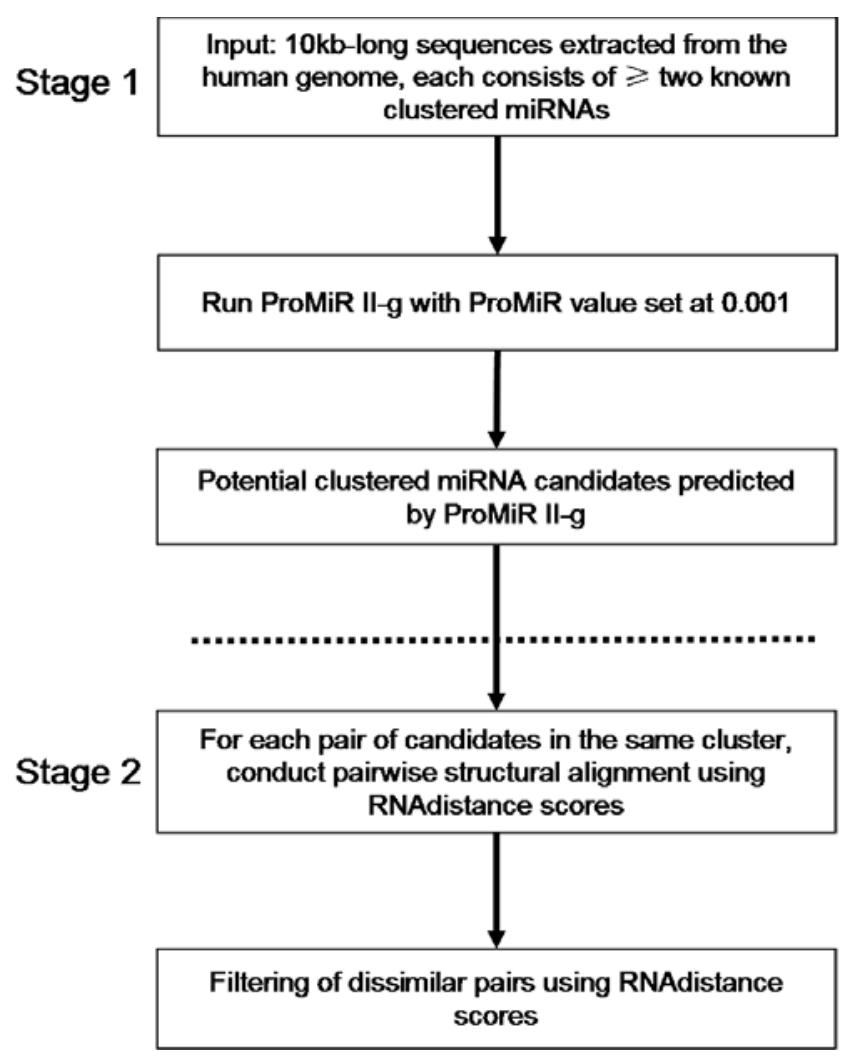

Figure 3

Overview of the clustering-based approach. In principle, our clustering-based approach consists of two stages. In stage I, a currently available prediction program, for example ProMirll-g, is selected to produce a list of potential candidates. A loose threshold is used because we want to include as many TPs as possible to achieve a high SE. In stage 2 , we aim at filtering the FPs from the list of candidates by picking out the dissimilar pairs as determined by the RNAdistance scores.

high pairwise RNAdistance scores with other candidates, it is likely to be a false positive and should be eliminated. The crucial step lies on how to determine candidate(s) with high scores and filtered them from the results. After several trials, we propose the following steps to do the filtering:

Step I

Calculate the lower quartile (LQ) score of all the pairwise RNAdistance scores formed by the candidates as the threshold. If a cluster has less than four candidates, the average score will be taken as the threshold.

Step 2

Select the potential candidates. Potential candidates are candidates which can form a pairwise score less than the threshold with another candidate, and these two candidates are regarded as a linked pair. For example, given that $\mathrm{c} 1$ and $\mathrm{c} 2$ are two of the candidates and $\mathrm{c}_{-}\{1,2\}$ denotes the RNAdistance score when they are aligned to each other, if the threshold score is 40 and $c_{-}\{1,2\}$ is $32, c_{1}$ and $c 2$ are potential candidates and linked pair.

Step 3

Adopt a brute-force approach and enumerate all combinations formed by the potential candidates with replacement. Only the combinations that are formed by linked pairs are of our interest. For example, if there is a combination of $\mathrm{c} 1, \mathrm{c} 2, \mathrm{c} 3, \mathrm{c} 4$ and $\mathrm{c} 5$, but $\mathrm{c} 1$ and $\mathrm{c} 5$ does not form a linked pair (i.e. $c_{-}\{1,5\}$ is less than the threshold), this combination will be discarded. Each combination is given a "R score" which is calculated as follows:

$\mathrm{R}_{-}\{1,2, \ldots, k-1, k\}=(1 / n)\left(\operatorname{sum}\left(\mathrm{c}_{-}\{1,2\}, \mathrm{c}_{-}\{1,3\}, \mathrm{c}_{-}\{1\right.\right.$, $\left.\left.4\}, c_{-}\{1, k\}, c_{-}\{2,3\}, c_{-}\{2,4\}, c_{-}\{2, k\}, \ldots ., c_{-}\{k-1, k\}\right)\right)$, where $k$ is the total number of candidates in the combination and $n$ is the combinatorial $k \mathrm{C} 2=k(k-1) / 2$

In simple terms, $\mathrm{R}$ score is calculated as the average of all the pairwise RNAdistance scores formed by the candidate pairs in the combination.

Step 4

Another threshold has to be determined using the R scores in order to select the final candidates from the combinations formed in Step 3.

If there is only one combination formed, the candidates which form this combination are taken as the results.

The R scores will be sorted in ascending order, e.g. R1, R2, $\mathrm{R} 3, \mathrm{R} 4, \ldots . . \mathrm{R} k$, where $k$ denotes the total number of combinations formed in Step 3. If $k$ is bigger than or equal to 3 and $(\mathrm{R} 3-\mathrm{R} 2)<(\mathrm{R} 2-\mathrm{R} 1)$, the threshold is taken as R3. Otherwise the threshold is taken as $\mathrm{R} 2$. If the threshold is less than 30, the threshold is set at 30 . Candidates which form a combination with a $\mathrm{R}$ score less than or equal to this threshold are the TPs and will be outputted as the answers.

\section{List of abbreviations used}

$\mathrm{FP}(\mathrm{s})$ : false positive(s); LQ: lower quartile; miRNA(s): microRNA(s); nt: nucleotides; PPV: positive predictive value; $\mathrm{SE}$ : sensitivity; $\mathrm{TP}(\mathrm{s})$ : true positive(s).

\section{Competing interests}

The authors declare that they have no competing interests.

\section{Authors' contributions}

WSL carried out the above studies and analyses, participated in the design of the study and drafted the manuscript. MCML and DWC revised the manuscript for important intellectual content. SMY participated in the 
Table 5: Results of our clustering-based approach on the human, mouse and rat genome data.

\begin{tabular}{|c|c|c|c|c|}
\hline & Human & Mouse (AII) & Mouse (Distinct) & Rat \\
\hline \# of predictions & 374 & 381 & 359 & 276 \\
\hline \# of TPs & 196 & 172 & 165 & 101 \\
\hline \# of real miRNAs & 240 & 245 & 212 & 146 \\
\hline SE & $81.67 \%$ & $70.20 \%$ & $77.83 \%$ & $69.18 \%$ \\
\hline Change in SE & $-7.91 \%$ & $-11.02 \%$ & $-8.09 \%$ & $-17.81 \%$ \\
\hline PPV & $52.41 \%$ & $45.14 \%$ & $45.96 \%$ & $36.59 \%$ \\
\hline Change in PPV & $+21.25 \%$ & $+14.81 \%$ & $+17.37 \%$ & $+15.94 \%$ \\
\hline
\end{tabular}

This table shows the SE and PPV obtained after our filtering approach is applied to the predicted candidates generated by ProMirll-g. The PPV is increased by more than $15 \%$ in all the three genomes. The SE is kept reasonably high as it is just slightly decreased by a percentage less than $10 \%$ in the human and mouse genomes.

design of the study and revised the manuscript. All authors read and approved the final manuscript.

\section{Acknowledgements}

The project is partially supported by the Seed Funding Programme for Basic Research of HKU (2006 I I I5900 I).

This article has been published as part of BMC Bioinformatics Volume 9 Supplement 12, 2008: Asia Pacific Bioinformatics Network (APBioNet) Seventh International Conference on Bioinformatics (InCoB2008). The full contents of the supplement are available online at http://www.biomedcentral.com/ $|47|-2 \mid 05 / 9$ ? issue $=\mathrm{S} \mid 2$.

\section{References}

I. Ambros V: The functions of animal microRNAs. Nature 2004, 43 I (7006):350-355.

2. Cullen BR: Viruses and microRNAs. Nat Genet 2006, 38(Suppl):S25-S30.

3. Griffiths-Jones S: The microRNA Registry. Nucleic Acids Res 2004:DI09-DIII.

4. Griffiths-Jones S, Grocock RJ, van Dongen S, Bateman A, Enright AJ: miRBase: microRNA sequences, targets and gene nomenclature. Nucleic Acids Res 2006:D I 40-DI44.

5. Bentwich I, Avniel A, Karov Y, Aharonov R, Gilad S, Barad O, Brazilai $A$, Einat $P$, Einav $U$, Meiri $E$, et al.: Identification of hundreds of conserved and nonconserved human microRNAs. Nat Genet 2005, 37(7):766-770.

6. Berezikov E, Guryev V, Belt J van de, Wienholds E, Plasterk RHA, Cuppen E: Phylogenetic shadowing and computational identification of human microRNA genes. Cell 2005, I 20(I):2I-24.

Table 6: Results of our clustering-based approach when applied on clusters with more than one TP.

\begin{tabular}{|c|c|c|c|c|c|c|c|c|}
\hline \multirow[b]{2}{*}{ Before/After filtering } & \multicolumn{2}{|c|}{ Human } & \multicolumn{2}{|c|}{ Mouse (AII) } & \multicolumn{2}{|c|}{ Mouse (Distinct) } & \multicolumn{2}{|c|}{ Rat } \\
\hline & before & after & before & after & before & after & before & after \\
\hline \# of predictions & 620 & 335 & 632 & 363 & 616 & 350 & 525 & 224 \\
\hline \# of TPs & 206 & 189 & 195 & 168 & 179 & 155 & 121 & 99 \\
\hline \# of real miRNAs & 220 & 220 & 234 & 234 & 202 & 202 & 132 & 132 \\
\hline SE & $93.64 \%$ & $85.91 \%$ & $83.33 \%$ & $71.79 \%$ & $88.61 \%$ & $76.73 \%$ & $91.67 \%$ & $75.00 \%$ \\
\hline Change in SE & - & $-7.73 \%$ & - & $-11.54 \%$ & - & $-11.88 \%$ & - & $-16.67 \%$ \\
\hline PPV & $33.23 \%$ & $56.42 \%$ & $30.85 \%$ & $46.28 \%$ & $29.06 \%$ & $44.29 \%$ & $23.05 \%$ & $44.20 \%$ \\
\hline Change in PPV & - & $+23.19 \%$ & - & $+15.43 \%$ & - & $+15.23 \%$ & - & $+21.15 \%$ \\
\hline
\end{tabular}

If we exclude the clusters which bear no TPs or just one TP among the candidates predicted by ProMirll-g, we can see a great improvement in PPV without a significant effect on SE after our filtering approach is applied. The results agree with the principle of our approach, which is developed based on the phenomenon of miRNA clustering. In other words, if there are no clustered miRNAs in a sequence, our approach is not going to work properly. This table presents the results of a fairer comparison, suggesting that our approach is effective in filtering FPs. 
7. Leung WS, Yiu SM, Cheung DW, Lai L, Lin MC, Kung HF: Computational prediction on mammalian and viral microRNAs - A review. IJIB 2007, I(2): I I8-I 26

8. Nam JW, Shin KR, Han J, Lee Y, Kim VN, Zhang BT: Human microRNA prediction through a probabilistic co-learning model of sequence and structure. Nucleic Acids Res 2005, 33( I I):3570-358I.

9. Nam JW, Kim J, Kim SK, Zhang BT: ProMiR II: a web server for the probabilistic prediction of clustered, nonclustered, conserved and nonconserved microRNAs. Nucleic Acids Res 2006:W455-W458.

10. Altuvia $Y$, Landgraf $P$, Lithwick G, Elefant N, Pfeffer S, Aravin A Brownstein MJ, Tuschl T, Margalit $\mathrm{H}$ : Clustering and conservation patterns of human microRNAs. Nucleic Acids Res 2005, 33:2697-2706

II. Tanzer A, Stadler PF: Molecular evolution of a microRNA cluster. J Mol Biol 2004, 339:327-335.

12. Tanzer A, Stadler PF: Evolution of microRNAs. Methods Mol Biol 2006, 342:335-350.

13. Hayashita $Y$, Osada H, Tatematsu $Y$, Yamada H, Yanagisawa K, Tomida S, Yatabe Y, Kawahara K, Sekido Y, Takahashi T: A polycistronic microRNA cluster, miR-17-92, is overexpressed in human lung cancers and enhances cell proliferation. Cancer Res 2005, 65:9628-9632.

14. Samols MA, Hu J, Skalsky RL, Renne R: Cloning and identification of a microRNA cluster within the latency-associated region of Kaposi's sarcoma-associated herpesvirus. J Virol 2005, 79:930I-9305.

15. Zhang R, Peng Y, Wang W, Su B: Rapid evolution of an X-linked microRNA cluster in primates. Genome Res 2007, 17:6/2-617.

16. Weber MJ: New human and mouse microRNA genes found by homology search. FEBS Journal 2005, 272(I):59-73.

17. Baskerville S, Bartel DP: Microarray profiling of microRNAs reveals frequent coexpression with neighboring miRNAs and host genes. RNA 2005, I I:24I-247.

18. Lagos-Quintana M, Rauhut R, Lendeckel W, Tuschl T: Identification of novel genes coding for small expressed RNAs. Science 200 I, 294(5543):853-858.

19. Lau NC, Lim LP, Weinstein EG, Bartel DP: An abundant class of tiny RNAs with probable regulatory roles in Caenorhabditis elegans. Science 200I, 294(5543):858-862.

20. Mourelatos Z, Dostie J, Paushkin S, Sharma A, Charroux B, Abel L, Rappsilber J, Mann M, Dreyfuss G: miRNPs: a novel class of ribonucleoproteins containing numerous microRNAs. Genes Dev 2002, 16:720-728.

2I. Hertel J, Lindemeyer M, Missal K, Fried C, Tanzer A, Flamm C, Hofacker IL, Stadler PF, 2005 SoBCLa: The expansion of the metazoan microRNA repertoire. BMC Genomics 2006, 7:25.

22. Notredame C, Higgins D, Heringa J: T-Coffee: A novel method for multiple sequence alignments. J Mol Biol 2000, 302:205-2I7.

23. Hofacker IL: Vienna RNA secondary structure server. Nucleic Acids Res 2003, 3 I ( I3):3429-343I.

24. Sewer A, Paul N, Landgraf P, Aravin A, Pfeffer S, Brownstein MJ, Tuschl T, van Nimwegen E, Zavolan M: Identification of clustered microRNAs using an $a b$ initio prediction method. BMC Bioinformatics 2005, 6:267.

25. Shapiro BA: An algorithm for comparing multiple RNA secondary structures. CABIOS 1988, 4(3):387-393.
Publish with Biomed Central and every scientist can read your work free of charge

\footnotetext{
"BioMed Central will be the most significant development for disseminating the results of biomedical research in our lifetime. " Sir Paul Nurse, Cancer Research UK

Your research papers will be:

- available free of charge to the entire biomedical community

- peer reviewed and published immediately upon acceptance

- cited in PubMed and archived on PubMed Central

- yours - you keep the copyright
}

Submit your manuscript here:

http://www.biomedcentral.com/info/publishing_adv.asp
BioMedcentral 